\title{
Switching diet from white to black rice can modulate the lipid profile and oxidative stress in rabbits?
}

\author{
A. R. Abdel-Moemin \\ Nutrition and Food Science Department, Faculty of Home Economics, Helwan University, Ain Helwan, 11795, Cairo, Egypt
}

Rice (Oryza sativa L.) is one of the most important crops that supply food for about half of the globe population. Egypt is one of the few countries that produce high-yielding rice varieties. The influence of white and black rice consumption on lipid profile, hydroperoxides, and thiobarbituirc reactive substances (TBARS) induced by hypercholesterolemia was investigated in rabbits. Twenty-four male rabbits were divided into four groups. They were fed a basal diet (control group, $n=6)$, a high-fat/cholesterol $(0.5 \mathrm{~g} / 100 \mathrm{~g})$ diet (HFC group, $n=6)$, a high-fat/cholesterol diet with $25 \mathrm{~g} / 100 \mathrm{~g}$ white ground rice (WGR group, $n=6$ ), and $25 \mathrm{~g} / 100 \mathrm{~g}$ black ground rice (BGR group, $n=6$ ) for 10 weeks. Blood samples were collected for lipid measurements and livers were removed for assessment of histological examination. Results indicate that serum HDL-C were higher $(P<0.05)$ in the BGR compared with the HFC and WGR groups. Hydroperoxides, TBARS, LDL, and VLDL were significantly lower in the BGR compared to WGR and HCF groups. In conclusion, black rice consumption was modulating the lipid profile and oxidative oxidation induced by dietary high fat and cholesterol. The enhanced serum HDL cholesterol and decreased hydroperoxides may be mechanisms of the cardioprotective effect of black rice. 